Classification

Physics Abstracts

$61.14 \mathrm{~F}-61.40-07.80$

\title{
Electron diffraction of amorphous thin films using PEELS
}

\author{
David Cockayne $\left({ }^{1}\right)$, David McKenzie $\left({ }^{2}\right)$ and David Muller $\left({ }^{1,2}\right)$ \\ ( ${ }^{1}$ ) Electron Microscope Unit, University of Sydney, NSW 2006, Australia \\ $\left(^{2}\right)$ School of Physics, University of Sydney, NSW 2006, Australia \\ (Received December 18, 1990; accepted March 26, 1991)
}

\begin{abstract}
In previous work, a method for obtaining accurate reduced density functions, $G(r)$, from chosen small regions of amorphous and polycrystalline thin films was described. The method involves scanning an electron diffraction pattern across the entrance aperture of a serial energy loss spectrometer. The method has been further developed, using a parallel energy loss spectrometer, and using filtering techniques to obtain more accurate data at both large and small scattering angles.
\end{abstract}

\section{Introduction.}

The use of a conventional transmission electron microscope for the collection of accurate diffraction intensity data from amorphous and polycrystalline specimens using a serial-type electron energy loss spectrometer [EELS] has been discussed in previous work [1]. Essentially the circularly symmetric diffraction pattern is scanned across the entrance aperture of an energy loss spectrometer under computer control with the energy loss window set to a fixed value, generally centred on zero loss, with full width at half maximum of a few $\mathrm{eV}$. The data set, which is the intensity $I(s)$ as a function of scattering angle $s=2 \sin (\theta) / \lambda$, is stored and manipulated in the multichannel analyser (MCA) in much the same way as for conventional energy loss spectrometry. The energy filtered diffraction pattern (EFDP) collected in this way is subsequently converted into a reduced density function, $G(r)$, using procedures discussed earlier [1]. The technique is a development of the earlier work of several other researchers (e.g. [2,3]). It gives accurate (to 1\%) nearest neighbour distances, and coordination numbers. The technique has been applied to a large number of structural problems in thin film physics (e.g. a-C [1], a-Si [4], a-Ge [5], and a variety of polycrystalline materials useful as optical or protective coatings such as $\mathrm{SiO}_{2} / \mathrm{TiO}_{2}$ [6], $\mathrm{ZrO}_{2}$ [7], c-BN [8], a-SiC:H [9]).

Despite the success of using the serial EELS (SEELS) as described above, a serious practical difficulty arose in that it proved difficult to ensure that the energy loss window did not drift during the collection time (typically five to ten minutes), which limited the accuracy of the results obtained, especially when determining coordination numbers. For this reason the technique has been converted to using a parallel EELS (PEELS) system, and this paper discusses its implementation and performance. 


\section{Instrumentation.}

The system used is a GATAN 666 PEELS, fitted beneath a Philips EM430 (300kV) TEM, interfaced to a TN5500 (and, more recently, to a Macintosh IIfx) which is used as an MCA. The PEELS has a voltage scan module (VSM) which applies an accurately calibrated voltage to the drift tube, under control from the MCA. The microscope is fitted with a $\mathrm{LaB}_{6}$ filament. Selected area diffraction patterns are obtained after adjusting the specimen height so that the specimen is in focus at a predetermined objective lens current. At the present time the minimum area that can be selected is limited by the need to have sufficient signal at high scattering angles.

The diffraction pattern is scanned across the entrance aperture of the spectrometer by means of a digitally incremented signal applied to the post-specimen scan coils of the TEM. In our case we have used the ramp available from the previous SEELS system, applied through the hybrid diffraction unit of the EM430, to perform this scan. However, any ramp generator which is matched to the scan coils of the microscope, and which can be operated under computer control, can be used. At each value of the ramp signal, corresponding to a particular $s$ value, an energy loss spectrum is collected by the MCA. In principle, each spectrum, corresponding to a different scattering angle $s$, could be stored for later processing. A block diagram of the system is shown in figure 1 .

For the case of the collection of the elastically scattered diffraction pattern, we integrate the zero loss peak within a defined energy loss window, and store the integrated values into consecutive channels of the MCA. The background can be removed either immediately after collection, or after collection of the entire diffraction pattern. In this way, the elastically scattered electron diffraction pattern is collected sequentially as a function of $s$.

\section{Energy filtering of spectra.}

The method described above for removing the background and integrating the zero loss peak can only be used for strong signals on uniform backgrounds. There are two effects which give difficulties. Firstly, at small scattering angles, the zero loss peak can become convolved with a strong plasmon. In this case, the background depends upon the scattering angle and cannot easily be removed after collection. Secondly, at large scattering angles, it is difficult to track the zero loss peak because of its low intensity compared with the noise. As the peak has a Gaussian profile, it is spread across many channels and, with fluctuations proportional to $1 \sqrt{N}$, at low count rates an error spike can easily be mistaken for the peak.

Both problems can be overcome by applying a smoothed second difference filter to the energy spectrum. Since differentiation is a linear operation, the overall shape of the diffraction pattern is preserved upon filtering. Using this procedure, not only are constant backgrounds removed, but any overlap with the plasmon is also removed. This follows because, far from the plasmon frequency, the plasmon peak has little curvature and hence has a small second derivative. In contrast, the zero loss peak is Gaussian in shape, and its second derivative has a narrow central peak compared with the width of the plasmon peak.

Figure 2 shows the effect of applying this procedure to the spectrum from a thin gold film at a small scattering angle where the plasmon is relatively large. It is seen that, after filtering, the plasmon is effectively stripped from the zero loss peak. The averaging properties of the top hat filter more than compensate for the counts lost to the side lobes (which broaden the peak overall).

Figure 3 shows the use of filtering to extract the zero loss peak from a noisy background, as occurs at large scattering angles.

Having applied the filter, only the channels between the zero crossings of the central peak are integrated, to avoid any catastrophic cancellation. This corresponds to integrating the Gaussian profile out to the FWHM. 

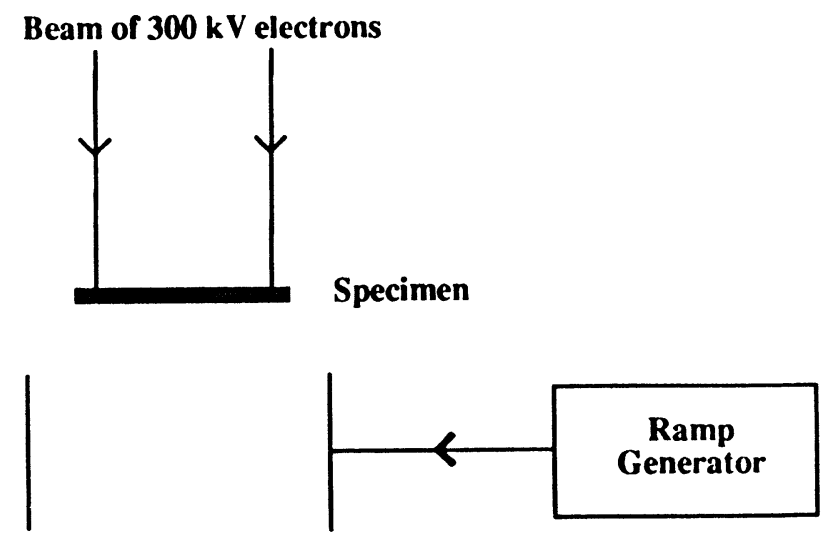

Moves Diffraction Pattern

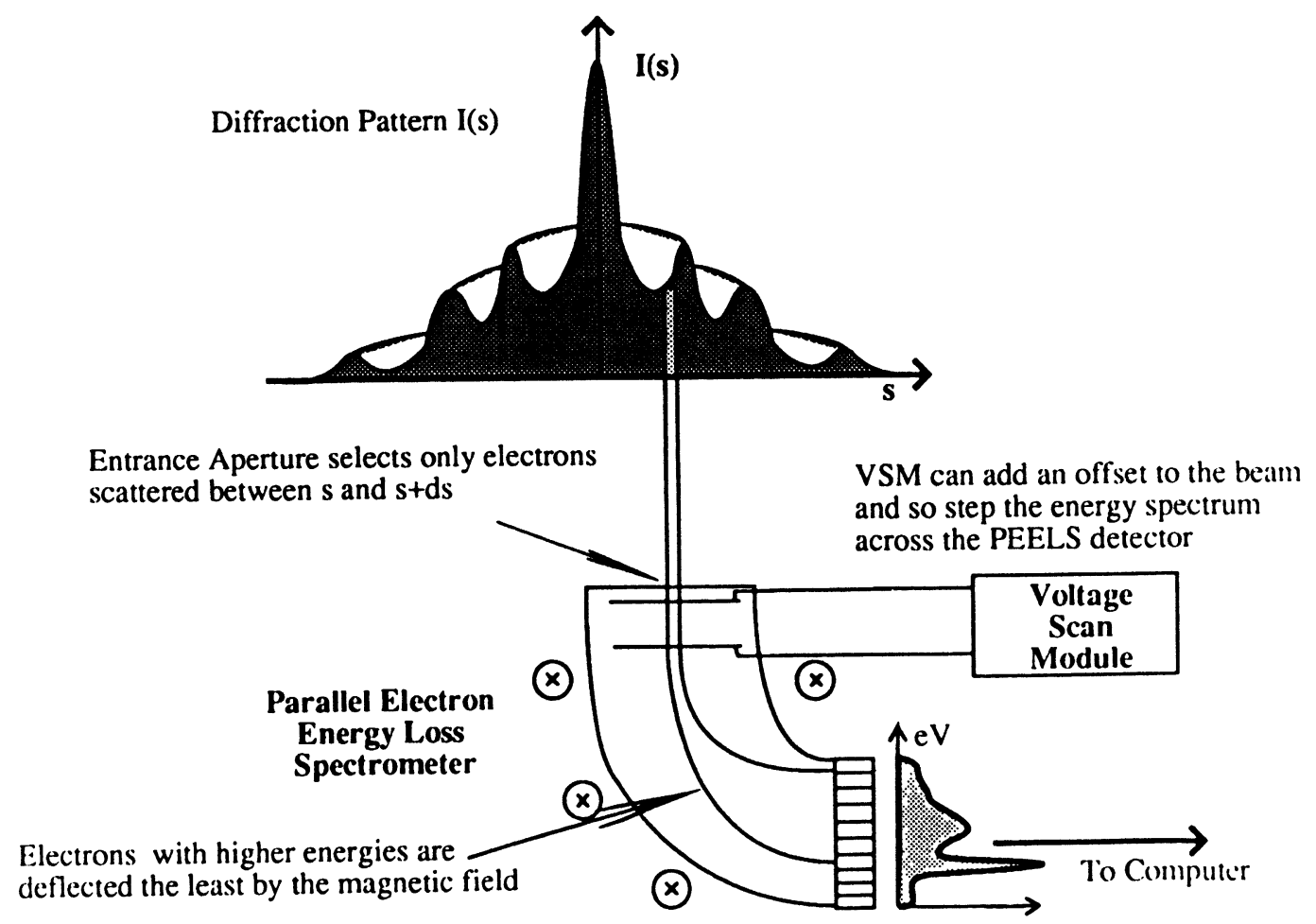

Fig. 1. - Block diagram of EFDP instrumentation using a PEELS. 


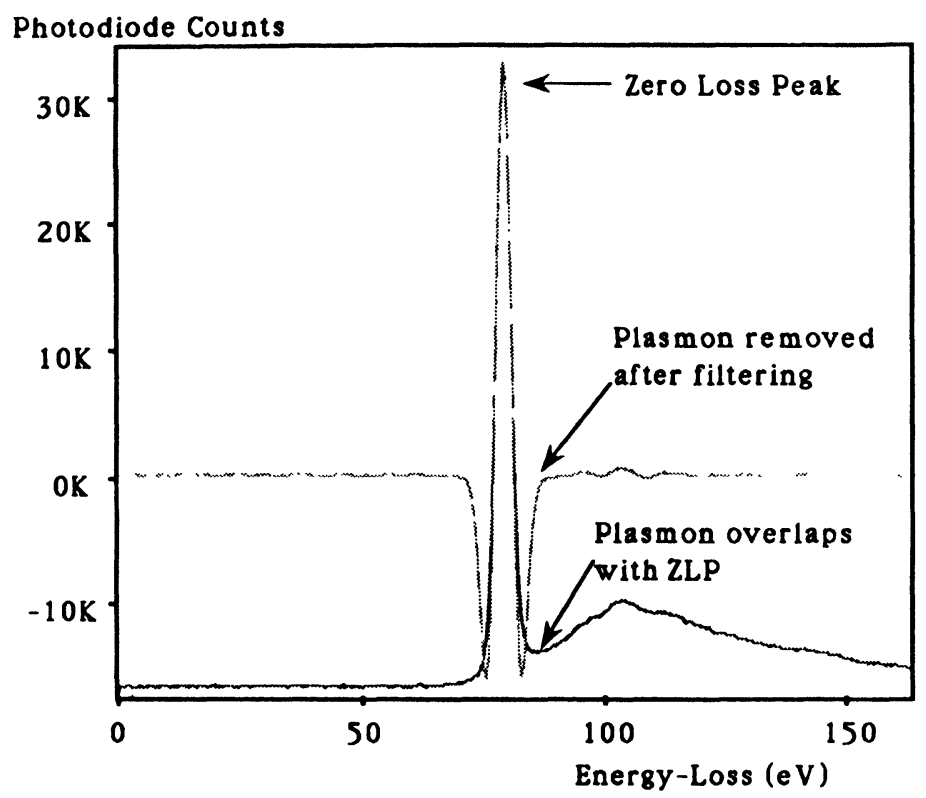

Fig. 2. - The zero loss peak at small $s$, before and after filtering, showing the extraction of the plasmon. The zero of the energy loss scale is arbitrary.

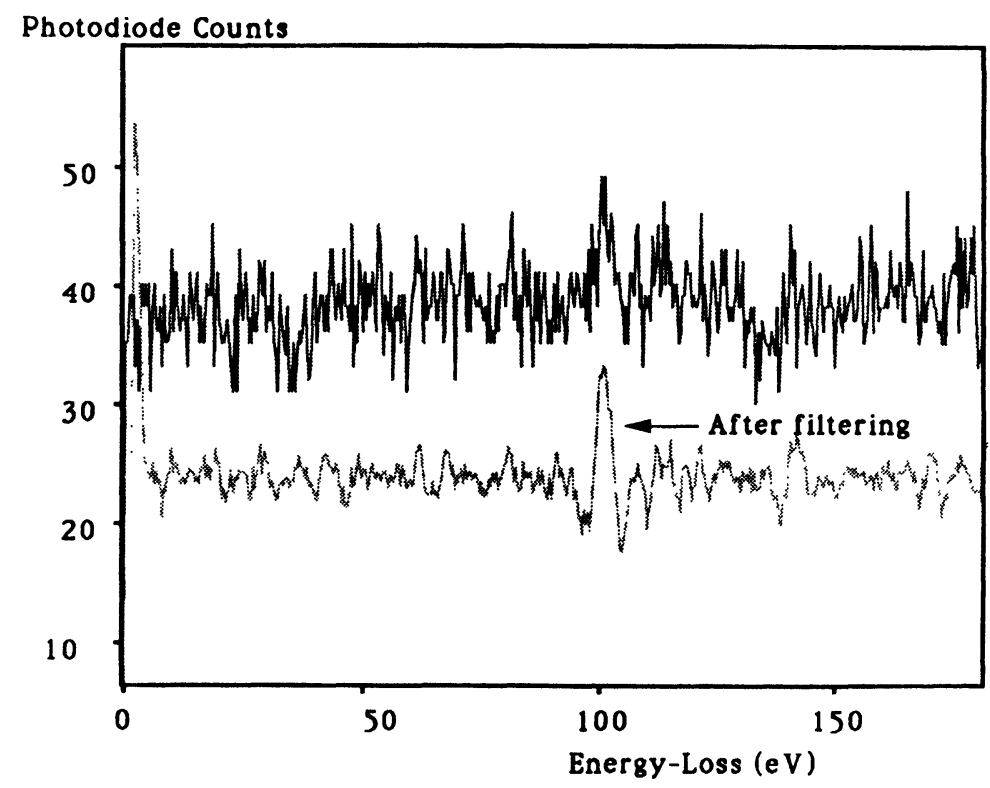

Fig. 3. - The use of filtering at high values of $s$ to reveal the zero loss peak. The zero of the energy loss scale is arbitrary. 


\section{Peculiarities of EFDP with PEELS.}

The electron intensity in the energy loss spectrum is obtained by measuring the stored charge remaining in a photodiode, after exposure to the light from the scintillator. The capacitor should be fully charged at the start of the collection interval. However, the characteristics of the GATAN 666 are such that incomplete charging occurs, resulting in an error in the measured signal. Moreover the degree of incomplete charging depends upon the extent of recharging required after the previous readout i.e. there is a "memory" effect. This effect, which is unimportant if numerous readouts of the same intensity level are made as in normal EELS analysis, can be important when recording varying signals, as occurs in this work. To overcome this problem, we use the VSM to shift the energy loss spectrum down in energy, so that the zero loss peak falls on diodes which have not been discharged (i.e. not been strongly illuminated) in previous readouts.

After a number of such increments of the VSM, translating the zero loss peak across the width of the diode array, the VSM is returned to its original value and the procedure is repeated. Figure 4 shows a set of four such readouts displayed together, after filtering.

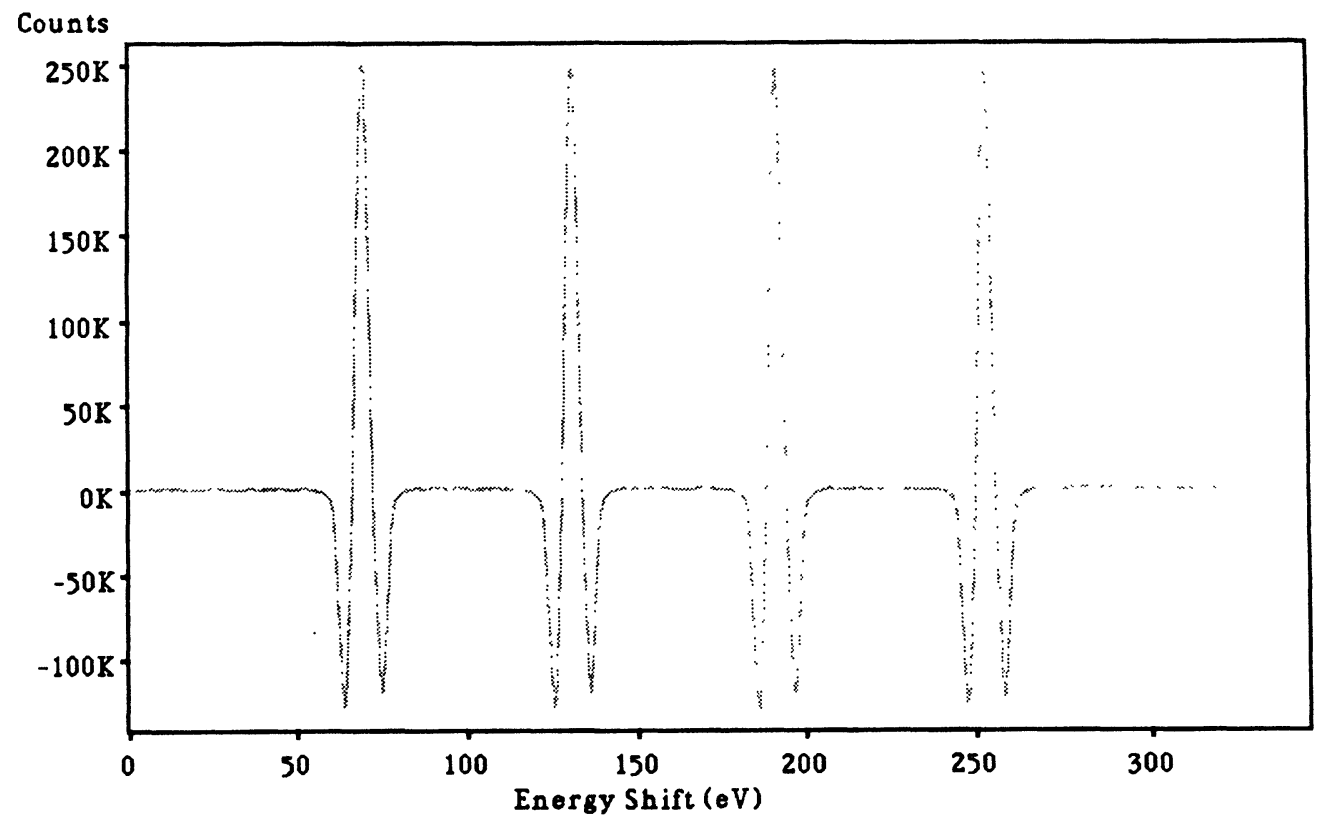

Fig. 4. - The display of four filtered zero loss peaks, displaced across the diode array using the VSM.

Whilst curing the "memory effect", this procedure introduces the difficulty that the five regions of the array used are not of equal sensitivity, resulting in a periodic variation in the recorded intensity, even for constant illumination. This variation is easily compensated for at the time of data processing. For greater accuracy in this correction, the same four sets of diodes can be used throughout, by making small adjustements to the VSM to compensate for any drift of the zero loss peak.

The electron intensities in a diffraction pattern can vary over several orders of magnitude, putting demands upon the dynamic range of the diode array. Experience has shown that care 
has to be taken not to exceed a maximum permissible count of $2^{14}-1$ in any one channel. This limit is overcome by summing a small number of identical collections, each with a maximum count less than this maximum, for each value of $s$.

At high scattering angles, the thermal and readout noise of the diodes can become comparable to the electron signal. The thermal noise is reduced by cooling the array using the built- in Peltier cooling system, but this is at the expense of increasing the "memory" effect referred to previously. The mean noise, together with the diode baseline, is removed using software procedures.

\section{Collection procedures.}

For accurate calibration of $G(r)$, it is essential that the angular scale of the diffraction pattern and its centre be accurately known. The angular scale of the diffraction pattern is determined by first collecting a diffraction pattern from a polycrystalline thin metal film ( $\mathrm{Au}$ or $\mathrm{Pt}$ ), as in figure 5. The collection proceeds from high to low $s$, where the upper limit to $s\left(s_{\max }\right)$ is set by the ability to distinguish the zero loss peak above background noise. This capability is enhanced by filtering, as described above. Typically $s_{\max }=4.8 \AA^{-1}$. The energy loss spectrum is collected for a preset readout time, and summed for a number of collections. A peak search routine centres the integration window about the zero-loss peak. The VSM steps the zero loss peak to a new region of the photodiode array, at the same time adjusting for any drift of the zero-loss peak detected during the previous peak search.

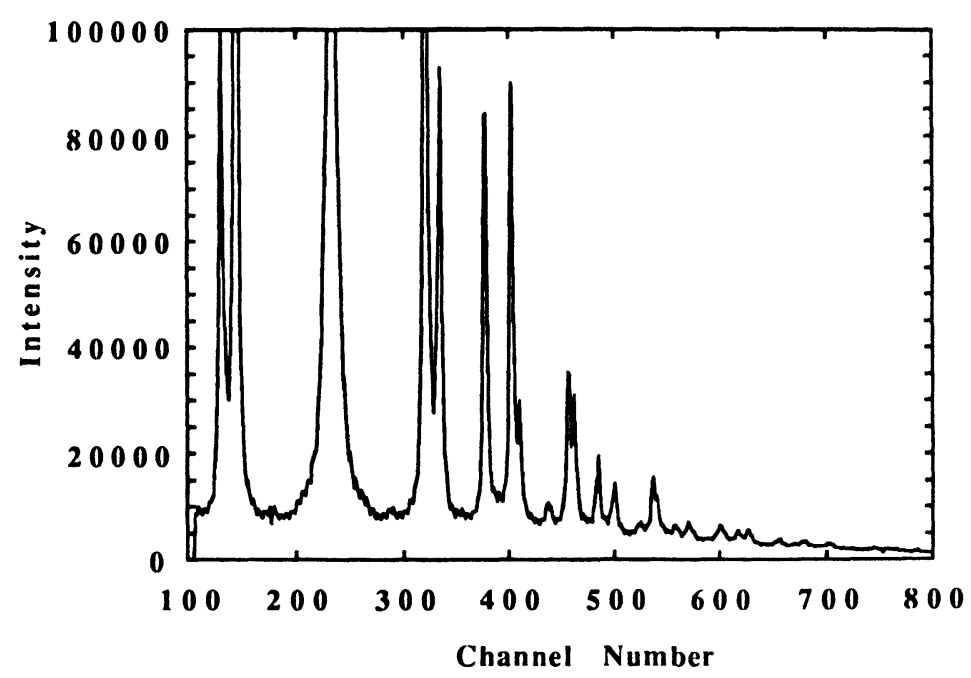

Fig. 5. - The electron diffraction pattern from a thin polycrystalline film of gold, as collected into the MCA.

This procedure is repeated for the full range of scattering angles, including scattering on both sides of the central spot of the diffraction pattern. This is necessary because the symmetry of the pattern is used to determine its centre. On passing through the central spot, the high electron intensity usually trips the overload protection, sending the GATAN 666 into the "attenuate" mode. At present, manual resetting is used when the intensity has dropped below the overload value. 
To collect the diffraction pattern from an amorphous or polycrystalline unknown specimen, the same procedure is followed, making sure that the electron optics remain as for the calibration spectrum.

The accuracy to which nearest neighbour distances can be determined is limited by several factors, including the accuracy with which the centre of the diffraction pattern can be established and the accuracy of the calibration of the scattering angle. While the value of $s_{\max }$ limits the resolution of $G(r)$, generally $I\left(s_{\max }\right)$ is relatively small, and the peaks in $G(r)$ are well separated. The accuracy to which the peak positions in $G(r)$ can be determined is then far greater than the resolution limit set by $s_{\max }$. Using typical experimental values of $s_{\max }$ of $4.8 \AA^{-1}$, it has been demonstrated [1] that nearest neighbour distances can be determined to an accuracy of $0.01 \AA$. This accuracy has been confirmed in many susequent studies.

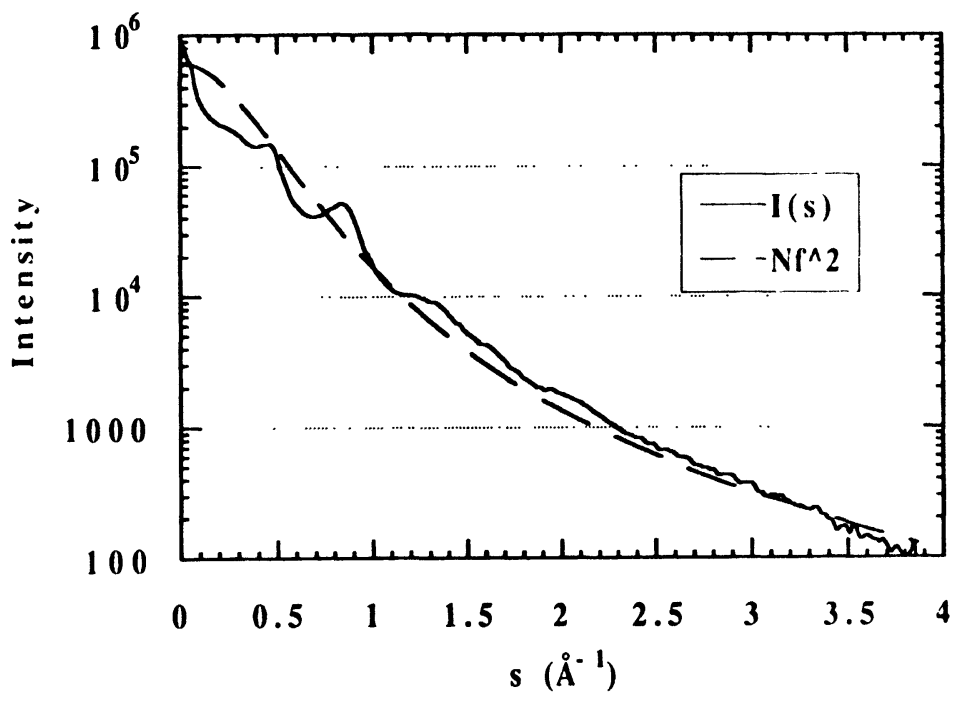

Fig. 6. $-I(s)$ from a-D:H displayed on a logarithmic scale, to show the fit to $N f^{2}$ out to $s=3.5 \AA^{-1}$.

As an example, figure 6 shows the diffraction pattern $I(s)$ from an amorphous hydrogenated diamond film, displayed on a logarithmic scale. It illustrates the excellent fit to $N f^{2}$ over a wide range of $s$. The resulting $G(r)$ is shown in figure 7. The nearest neighbour distance of $1.53 \pm 0.01 \AA$ and bond angle of $110^{\circ} \pm 1^{\circ}$ (determined from first and second nearest neighbour distances) show conclusively that the specimen consists almost entirely of $\mathrm{sp}^{3}$ hybridised carbon.

Advantages of this collection procedure over the SEELS procedure are:

1. drift of the zero loss peak is fully compensated for, allowing unattended collection;

2. the complete energy loss spectrum is available at each scattering angle, which, for example, will allow the study of plasmon dispersion;

3. extraction of the zero loss peak from overlapping inelastically scattered electrons can be achieved, giving more accurate $I(s)$ especially at small $s$.

Preliminary experiments using an amorphous carbon specimen and a small (20nm) approximately parallel probe in the nanoprobe mode show that useful data can be collected to $s=2 \AA^{-1}$ even for such a small electron probe. 


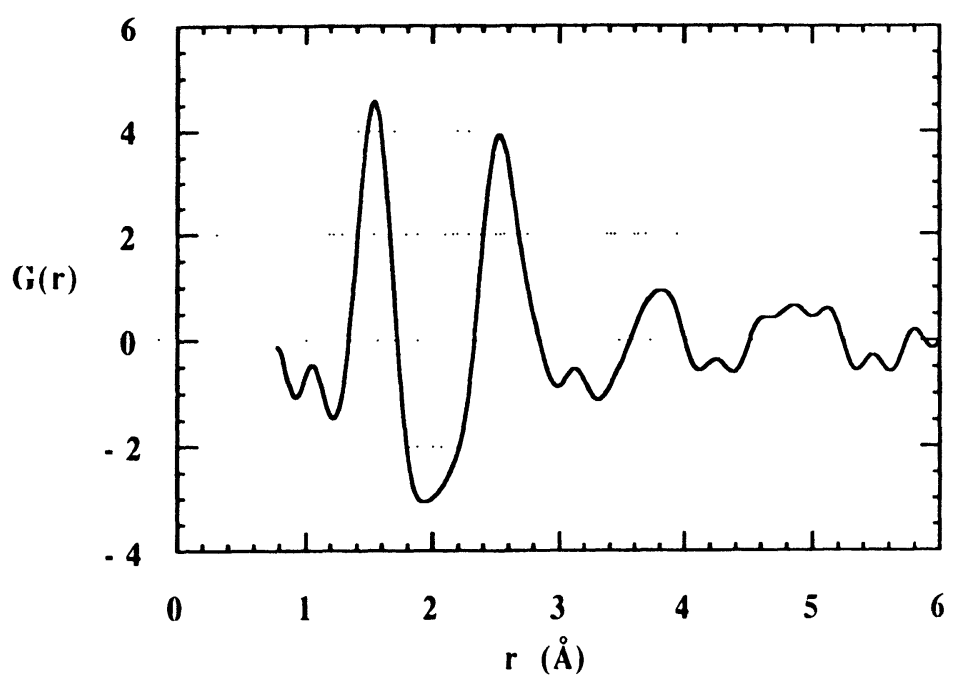

Fig. 7. $-G(r)$ obtained directly from the data of figure 6.

\section{Conclusion.}

The conversion of the technique of collecting the EFDP (energy filtered diffraction pattern) to a PEELS system has allowed the sensitivity and accuracy of the technique to be appreciably refined. Collections of $I(s)$ and conversions to $G(r)$ within 10 minutes are now feasible, which provides a technique useful for on-line analysis.

\section{Acknowledgements.}

We are grateful to Dr Ondrej Krivanek and Staff of GATAN for most helpful advice and comments on development aspects of this work.

\section{References}

[1] COCKAYNE D.J.H. and MCKEnZIE D.R., Acta. Cryst. A44 (1988) 870.

[2] GRIGSON C.W.B., J. Electron. Control 12 (1962) 209.

[3] GracsYK J.F. and Moss S.C., Rev. Sci. Instrum. 40 (1969) 424.

[4] Liu Z.Q., MCKenZIE D.R., COCKAYNE D.J.H. and DwarTe D.M., Phil. Mag. B 57 (1988) 753.

[5] WhITE S.B., Ph D Thesis, University of Sydney (1988).

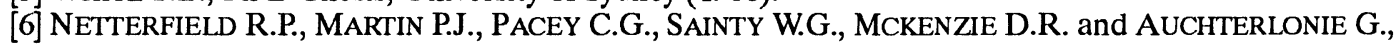
J. Appl. Phys. 66 (1989) 1805.

[7] MCKENZIE D.R., COCKAYNE D.J.H., SCEATS M.G., MARTIN P.J., SAINTY W.G. and NETTERFIELD R.P., J. Mater. Sci. 22 (1987) 3725.

[8] MCKENZIE D.R., MULLER D. and COCKAYNE D.J.H. (in preparation).

[9] MCKEnZIE D.R., SMIth G.B. and LiU Z.Q., Phys. Rev. B 37 (1988) 8875. 